\title{
Naked Nuclei Revisited: p63 Immunoexpression
}

\author{
Jorge S. Reis-Filho, M.D. ${ }^{1,2}$ Andre Albergaria, B.M., ${ }^{1}$ \\ Fernanda Milanezi, M.D., ${ }^{1}$ Isabel Amendoeira, M.D., ${ }^{3}$ \\ and Fernando C. Schmitt, M.D., Ph.D., M.I.A.C. ${ }^{1,4^{\prime} *}$
}

\begin{abstract}
The presence of naked nuclei $(N N)$ in cytological preparations of fibroadenomas is a well-known finding. Regardless of their importance on the differential diagnosis of fibroadenoma and other benign lesions of the breast, the origin of $N N$ remains elusive. Despite previous efforts to characterize them, the lack of a reliable nuclear marker for myoepithelial cells impaired definitive conclusions. We performed a systematic evaluation of p63 expression in cytological and histological preparations of 10 fibroadenoma specimens. We observed that in histological sections, p63 was restricted to the nuclei of myoepithelial/basal cells in lobules and ducts of normal breast. In fibroadenomas, p63 decorated the nuclei of myoepithelial cells in the periphery of epithelial duct-like formations and slit-like formations. No p63 immunoreactivity was observed in stromal or epithelial cells. In cytological samples, almost all NN and cells surrounding epithelial cell clusters were stained; no stromal cell admixed with fibrillary matrix or epithelial cell was stained with p63. Based on our findings, we strongly suggest that most, if not all, NN are myoepithelial in origin. Diagn. Cytopathol. 2002;27:
\end{abstract} 135-138. ๑ 2002 Wiley-Liss, Inc.

Key Words: fibroadenoma; p63; myoepithelial cell; stromal cell; naked nuclei

The presence of naked nuclei $(\mathrm{NN})$ is one of the most important criteria to support a diagnosis of benign lesion in fine needle aspiration biopsies (FNABs) of the breast. ${ }^{1,2,3}$ Generally, the presence of a large number of NN points toward a diagnosis of benignity. In breast cytology NN are especially observed in fibroadenomas. ${ }^{1-3}$

${ }^{1}$ IPATIMUP - Institute of Molecular Pathology and Immunology, University of Porto, Porto, Portugal

${ }^{2}$ School of Health Sciences, University of Minho, Braga, Portugal

${ }^{3}$ Hospital São João, University of Porto, Porto, Portugal

${ }^{4}$ Medical Faculty, University of Porto, Porto, Portugal

Grant sponsor: Fundação para a Ciência e Tecnologia, Portugal; Grant number: SFRH/BD/5386/2001.

*Correspondence to: Fernando C. Schmitt, M.D., Ph.D., M.I.A.C., IPATIMUP, R. Roberto Frias, S/N, 4200, Porto, Portugal.

E-mail: fernando.schmitt@ipatimup.pt

Received 15 February 2002; Accepted 17 March 2002

DOI 10.1002/dc.10164

Published online in Wiley InterScience (www.interscience.wiley.com).
Regardless of their important role in the diagnosis of benign lesions, the origin of $\mathrm{NN}$ remains disputable. ${ }^{4}$ Most authors claim that NN derive from myoepithelial cells; $5,6,7$ however, Tsuchiya et al., ${ }^{4}$ based on ultrastructural features, suggested a stromal origin for these cells. This issue is far from being resolved, mainly due to methodological difficulties to address the question because no reliable nuclear myoepithelial cell marker was described until the last year.

P63, a recently characterized p53 homolog, is consistently expressed in basal cells of several types of multilayered epithelia and in myoepithelial cells of the breast and sweat glands. ${ }^{8,9}$ It has been shown that p63 also decorates the nuclei of myoepithelial cells in normal breast lobules and ducts, as well as in several types of myoepithelialderived tumors. ${ }^{9}$ In a brief assessment of p63 distribution in cytologic specimens, Barbareschi et al. ${ }^{9}$ demonstrated that $\mathrm{NN}$ consistently expressed p63. We evaluated the immunoreactivity of p63, a novel myoepithelial/basal cell nuclear immunohistochemical marker, in NNs on cytologic and histologic samples of fibroadenomas.

\section{Material and Methods}

\section{Cytological Samples}

Cases of fibroadenomas diagnosed by fine-needle aspiration biopsy were retrieved from the files of the Pathology Department, Hospital São João, Porto, Portugal, and IPATIMUP, Porto, Portugal. Only those cases in which alcoholfixed Papanicolaou-stained slides and their respective histological resections were available were included in the present study.

These cases were independently reviewed by four pathologists, who searched for the most representative samples that were defined as: 1) samples that showed high cellularity; 2) were composed of large and branching monolayered sheets and antler-like papillary fronds of fairly uniform epithelial cells; 3) fragments of fibromyxoid stroma; 4) a large quantity of NN. Neither atypical epithelial cells nor adipocytes and inflammatory cells admixed with stromal fragments were observed. In only 10 specimens was a 
consensus reached among all pathologists regarding all of the above-cited criteria. Only these cases were included in the present study.

\section{Histological Samples}

Ten cases of fibroadenoma were formalin-fixed and embedded in paraffin. Four- $\mu \mathrm{m}$ histological sections were cut and routinely stained with $\mathrm{H} \& \mathrm{E}$. At histological examination, all cases showed the prototypical histological features of fibroadenoma. Serial sections of the most representative areas were cut at $4 \mu \mathrm{m}$ and mounted on silane-coated slides for immunohistochemistry.

\section{Immunocytochemistry and Immunohistochemistry}

The coverglass of previously Papanicolaou-stained smears was removed with immersion in xylene for $48 \mathrm{hr}$. After washing with tap water, wet bath heat-induced antigen retrieval was made using Dako Antigen Retrieval Solution (Dako, Glostrup, Denmark) for $20 \mathrm{~min}$, followed by the streptavidin-biotin-peroxidase technique using antibodies raised against p63 (clone 4A4, dilution 1:150; Neomarkers, Freemont, CA, USA) as described elsewhere. ${ }^{9,10}$ The formalin-fixed, paraffin-embedded breast tissues were immunostained by p63 using the same protocol on cut histological sections mounted on silane-coated slides. Only cells showing strong and distinctive nuclear immunoreactivity for p63 were considered positive. Cytoplasmic and membranous staining were considered nonspecific. Positive and negative controls were included in each staining run.

\section{Results}

\section{Histological and Immunohistochemical Analysis}

On histological examination, all cases showed the typical histological appearance of fibroadenomas, including a loose stromal component, composed of bland spindle-shaped cells immersed in a variably collagenized fibromyxoid matrix and an epithelial component composed of two-layered epithelial-myoepithelial ductular structures and slit-like spaces.

In all cases immunohistochemistry showed a rather remarkable nuclear reactivity for p63 in the nuclei of myoepithelial cells located between epithelial cells and the stromal component of fibroadenomas (Fig. C-1A). In the adjacent breast tissue, p63 was also confined to the nuclei of myoepithelial/basal cells of breast lobules and ducts (Fig. C-1B). No p63-positive stromal cells, endothelial cells, or epithelial cells were observed in any specimens evaluated.

Based on these findings and on those previously reported by Barbareschi et al., ${ }^{9}$ we concluded that p63 was restricted to the myoepithelial/basal cell component.

\section{Cytological and Immunocytochemical Findings}

All samples showed highly cellular smears and were composed of an admixture of cohesive epithelial cell clusters, fragments of fibromyxoid stroma, isolated cells, and NN.
The epithelial cell clusters and sheets were arranged in monolayers with either a honeycomb or a antler-horn or finger-like pattern and consisted of cells with moderate-toabundant cytoplasm, well-defined cytoplasmic borders, round-to-oval nuclei, with discrete nucleoli. Oval-to-spindle-shaped cells with ill-defined cytoplasm and dark-stained nuclei were arranged on the periphery of epithelial cell clusters. Fragments of fibromyxoid stroma composed of spindle-shaped cells with hardly discernible cytoplasm immersed in a pink or blue-gray fibrillary matrix were seen in all cases. A variable number of isolated cells with oval nuclei and a sparse-to-moderate oval-to-spindle-shaped cytoplasm were observed. Small cells with oval naked nuclei were seen lying free in the background between epithelial cell clusters and fragments of fibromyxoid stroma.

In accordance with the immunohistochemical findings, immunocytochemistry with p63 antibody highlighted the nuclei of two distinct cell populations: 1) all of the oval-tospindle-shaped cells with dark nuclei on epithelial cell clusters (Fig. C-2A), and 2) all of the naked nuclei observed in the background of the smears (Fig. C-2B and inset). No immunoreactivity for p63 was observed in cells admixed with fibrillary matrix of fibromyxoid stroma or in those isolated cells with oval nuclei and sparse-to-moderate cytoplasm. Concordant results were observed in all specimens.

In accordance with histological and immunohistochemical findings, as well as based on the cytomorphological appearance of the p63-postive cells, we concluded that these cells were of myoepithelial origin.

\section{Discussion}

p63, a p53 homolog, is a protein necessary for the maintenance of a basal compartment of several multilayered epithelia. $^{8,9}$ The TP63 gene, located on $3 q 27$, encodes six different subtypes of transcripts, three with transactivating (TA-p63) properties and three truncated isoforms (DNp63). ${ }^{8,9}$ While TA-p63 is able to transactivate p53 reporter genes, DN-p63 act as a dominant negative for them. ${ }^{8,9}$ Interestingly, p63 does not seem to be a tumor-suppressor gene; indeed, p63 germline mutations are associated with limb-mammary syndrome, Hay-Wells syndrome, and ectrodactily, ectodermal dysplasia, and facial clefts (EEC) syndrome. ${ }^{8,9}$ p63 knockout mice show severe defects in epithelial development and cranial structures; interestingly, mammary glands are lacking in these mice, demonstrating the importance of p63 in mammary gland development. ${ }^{8,9}$ Recently, Barbareschi et al. ${ }^{9}$ described p63 immunoreactivity on histological sections in the nuclei of myoepithelial and basal cells of normal breast, as well as in the myoepithelial component of fibroadenomas, adenomyoepitheliomas, adenoid-cystic carcinomas, and in $4.6 \%$ of ductal carcinomas of the breast. ${ }^{9}$ Moreover, these authors briefly reported p63 immunoexpression on five cytological samples 

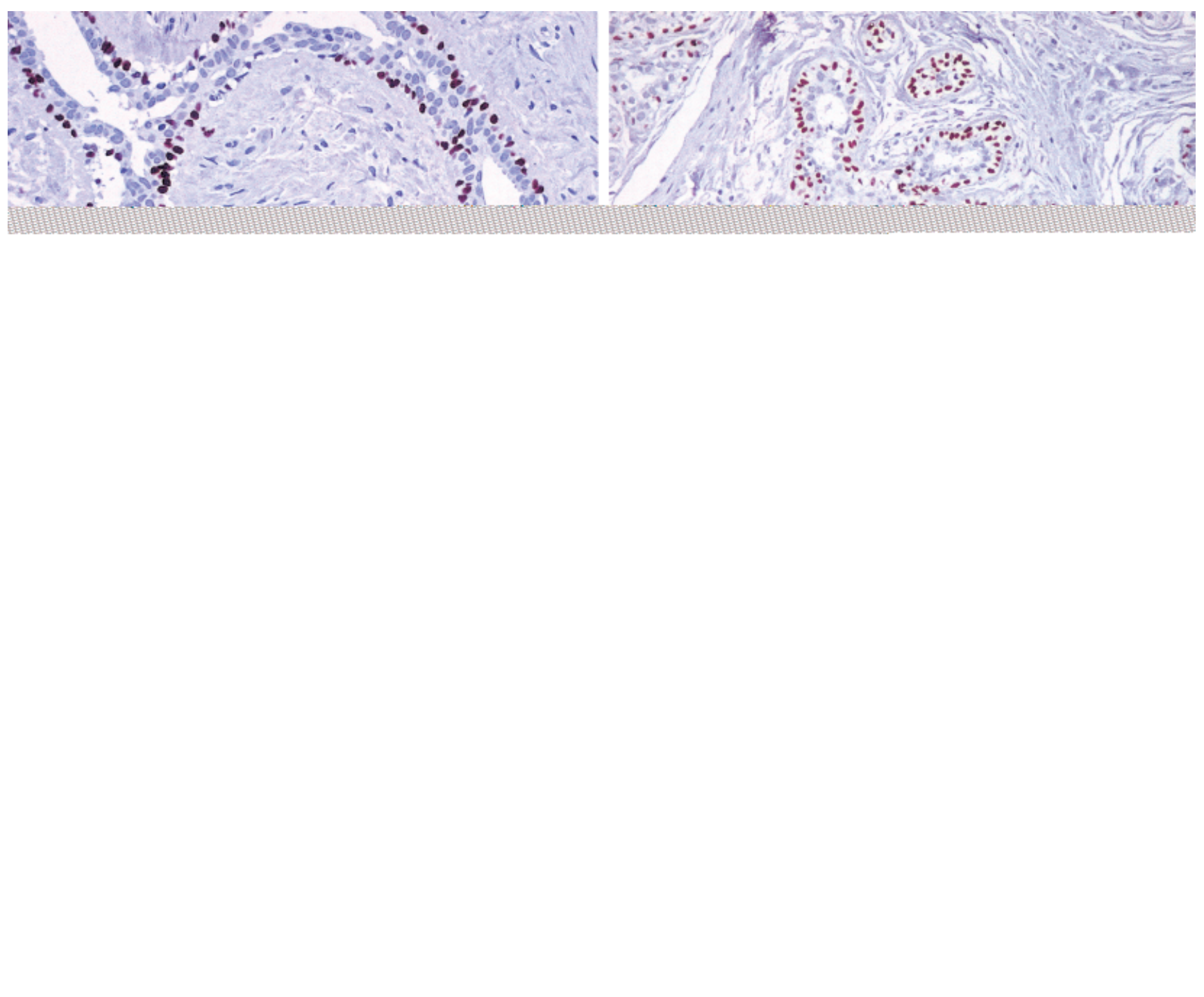

Fig. C-1. A: p63 immunoexpression in the nuclei of myoepithelial cells of a fibroadenoma. Note that there are no stromal or epithelial cells stained with this antibody (streptavidin-biotin-peroxidase $\times 200$ ). B: p63 expression in an adjacent breast lobule: only myoepithelial cells show nuclear reactivity for this antibody (streptavidin-biotin-peroxidase $\times 100$ )

Fig. C-2. A: Smear of a fibroadenoma showing a typical monolayered cell cluster. Note the distinctive p63 nuclear immunoreactivity in cells with morphological features compatible with myoepithelial cells (streptavidin-biotin-peroxidase $\times 100$ ). B: Medium-power-magnification showing an epithelialmyoepithelial cell cluster in the center of the field. Note that cells with myoepithelial cell morphology and almost all naked nuclei exhibit distinctive p63-immunoreactivity (streptavidin-biotin-peroxidase $\times 200$ ). Inset: Detail of NN with strong p63-immunoreactivity (streptavidin-biotin-peroxidase $\times 400$ ).

of fibroadenoma. ${ }^{9}$ In all of them almost all of the NN showed strong p63 immunoreactivity. ${ }^{9}$

$\mathrm{NN}$ are an important criterion in the diagnosis of fibroadenoma, ${ }^{1,2,3}$ Phyllodes tumor, ${ }^{11,12}$ fibrocystic mastopathy, ${ }^{1}$ pseudoangiomatous stromal hyperplasia, ${ }^{13}$ sclerosing lobular hyperplasia, ${ }^{14}$ benign papillary lesions, ${ }^{1,15}$ papillary adenoma of the nipple, ${ }^{15}$ gynecomastia, ${ }^{16}$ and other benign diseases. Occasionally, they may also be observed in aspirates from breast carcinomas. ${ }^{2,3}$ The presence of $\mathrm{NN}$ is a well-known diagnostic criterion that indicates the possibility of a benign lesion; in the series reported by Linsk et al., ${ }^{2}$ NN were observed in $25.2 \%$ (53 of 210 fibroadenomas), but only rarely in carcinomas; in contrast, Bottles et al. ${ }^{3}$ de- scribed the presence of $\mathrm{NN}$ in $91.9 \%$ of fibroadenoma smears, $88.1 \%$ of fibrocystic smears, and $20 \%$ of ductal carcinoma smears.

Regardless of their high prevalence in smears of fibroadenomas and other benign lesions, the origin of $\mathrm{NN}$ is still disputable. ${ }^{2,4-7}$ Based on microscopic evaluations, Zajicek et al. ${ }^{6}$ and Linsk et al. ${ }^{2}$ suggested a myoepithelial origin for these cells, which was corroborated by the ultrastructural analysis done by Ferguson et al., ${ }^{7}$ who also suggested that in FNAB the extraction process might result in a shearing between the epithelium and basal lamina, leading to myoepithelial cell lysis. On the other hand, Tsuchiya et al. ${ }^{4}$ suggested that NN were indeed derived from stromal cells, 
based on the lack of ATPase expression (a putative myoepithelial marker) and on the presence of ultrastructural features that are characteristically observed in stromal cells, such as abundant rough endoplasmic reticulum, numerous ribosomes, and lack of cytoplasmic filaments and dense bodies. ${ }^{4}$ Interestingly, Tsuchiya et al. $^{4}$ adopted a rather surprising criteria to define $\mathrm{NN}$; these authors stated that there were two types of NN: 1) round, darkly stained nuclei with compact chromatin and no cytoplasm; and 2) oval nuclei with a sparse-to-moderate cytoplasm. It should be stressed that the second type was the only type evaluated in the study performed by Tsuchiya et al. ${ }^{4}$ In addition, those authors also state that cells with round, darkly stained nuclei with compact chromatin remained with an uncertain histogenesis, since neither ATPase cytoplasmic immunoreactivity nor ultrastructural studies could provide insight on their origin. ${ }^{4}$ In our view, only cells of the first type are true NN, because cells of the second type show a small but distinctive cytoplasm.

Recently, this difficulty in the definition of NN origin has been overcome. Barbareschi et al. ${ }^{9}$ described p63 immunoreactivity in the nuclei of myoepithelial cells in normal breast, benign lesions, and malignant tumors with myoepithelial histogenesis. Moreover, they briefly illustrated that almost all NN were p63 immunoreactive. ${ }^{9}$ We expanded this observation in our 10 cases of fibroadenomas, in which p63 decorated nuclei of myoepithelial cells and failed to stain stromal cells on histological sections. Moreover, our cytological findings are in total agreement with those reported by Barbareschi et al., ${ }^{9}$ who reported intense p63 immunoreactivity in NN and in a subset of cells surrounding the epithelial cell clusters in cytoplasmic preparations of fibroadenoma.

Based on our results and those previously reported, ${ }^{9}$ we strongly suggest that in fibroadenoma samples the majority of NN, defined as oval-to-spindle-shaped cells without any discernible cytoplasm, show a myoepithelial origin. Thus, they might be included within the major criteria to diagnose benign breast lesions. Moreover, p63 seems to be an efficient ancillary method to identify myoepithelial cells and might play a major role in the identification of these cells in sparsely cellular or dubious cases. Further studies are warranted to evaluate the diagnostic and prognostic implications of p63 in a large series of aspirates from breast lesions.

\section{Acknowledgment}

Jorge S. Reis-Filho is a recipient of a Ph.D. grant from Portuguese Science and Technology Foundation.

\section{References}

1. Silverman JF. Breast. In: Bibbo M. Comprehensive cytopathology, 2nd ed. Philadelphia: W.B. Saunders; 1997. p 731-780

2. Linsk J, Kreuzer G, Zajicek J. Cytological diagnosis of mammary tumors from aspiration biopsies smears. II. Studies on 210 fibroadenomas and 210 cases of benign dysplasia. Acta Cytol 1972;16:130 138 .

3. Bottles K, Chan JS, Holly EA, Chiu SH, Miller TR. Cytologic criteria for fibroadenoma. A step-wise logistic regression analysis. Am J Clin Pathol 1988;89:707-713.

4. Tsuchiya S, Maruyama Y, Koike Y, Yamada K, Kobayashi Y, Kagaya A. Cytologic characteristics and origin of naked nuclei in breast aspirate smears. Acta Cytol 1987;31:285-290.

5. Marasa L, Tomasino RM. Aspiration cytology of the breast. II. Significance of bipolar naked nuclei. Pathologica 1982;74:193-200.

6. Zajicek J, Caspersson T, Jakobsson P, Kudinowski J, Linsk J, UsKrasovec M. Cytological diagnosis of mammary tumors from aspiration biopsy smears: comparison of cytological and histological findings in 2111 lesions and diagnostic use of cytophotometry. Acta Cytol 1970;14:370-376.

7. Ferguson DJ, Wells CA, Crucioli V. Ultrastructural analysis of fine needle aspirates from benign breast lesions. J Clin Pathol 1990;43:2226

8. Reis-Filho JS, Schmitt FC. Taking advantage of basic research: p63 is a reliable myoepithelial and stem cell marker. Adv Anat Pathol 2002; in press.

9. Barbareschi M, Pecciarini L, Cangi MG, et al. p63, a p53 homologue, is a selective nuclear marker of myoepithelial cells of the human breast. Am J Surg Pathol 2001;25:1054-1060.

10. Schmitt FC, Mera A. Fine needle aspiration cytology presentation of a cellular variant of breast myofibroblastoma. Report of a case with immunohistochemical studies. Acta Cytol 1998;42:721-724.

11. Simi U, Moretti D, Iacconi P, et al. Fine needle aspiration cytopathology of phyllodes tumor. Differential diagnosis with fibroadenoma. Acta Cytol 1988;32:63-66.

12. Shet T, Rege J. Cystic degeneration in phyllodes tumor. A source of error in cytologic interpretation. Acta Cytol 2000;44:163-168.

13. Vicandi B, Jimenez-Heffernan JA, Lopez-Ferrer P, Ortega L, Viguer JM. Nodular pseudoangiomatous stromal hyperplasia of the breast. Cytologic features. Acta Cytol 1998;42:335-341.

14. Jain M, Arora VK, Singh N, Bhatia A. Fine needle aspiration cytology of sclerosing lobular hyperplasia of the breast: a case report. Acta Cytol 2001;45:765-767.

15. Sood N, Jayaram G. Cytology of papillary adenoma of the nipple: a case diagnosed on fine-needle aspiration. Diagn Cytopathol 1990;6: 345-348.

16. Russin VL, Lachowicz C, Kline TS. Male breast lesions: gynecomastia and its distinction from carcinoma by aspiration biopsy cytology. Diagn Cytopathol 1989;5:243-247. 